\title{
Magnetostriction in Amorphous Co66Fe34 Microcantilevers Fabricated with Hydrogenated Amorphous Silicon
}

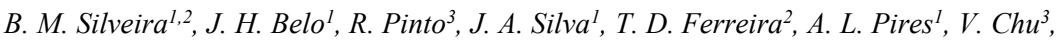 \\ J. P. Conde ${ }^{3,4}$, O. Frazão ${ }^{2}$, and A. M. Pereira ${ }^{1 *}$ \\ ${ }^{1}$ IFIMUP-IN - Institute for Nanoscience and Nanotechnology, Rua do Campo Alegre, 4169-007, Porto, Portugal \\ ${ }^{2}$ INESC-TEC - Technology and Science, Rua do Campo Alegre, 4169-007, Porto, Portugal \\ ${ }^{3}$ INESC-MN - Microsystems and Nanotechnologies, Rua Alves Redol, 1000-029 Lisboa, Portugal \\ ${ }^{4}$ Department of Bioengineering, Instituto Superior Técnico, Universidade de Lisboa, 1049-001 Lisboa, Portugal
}

\begin{abstract}
To study the magnetostriction of $\mathrm{Co}_{66} \mathrm{Fe}_{34}$ thin films, amorphous silicon microcantilevers were prepared by surface micromachining, and the $136 \mathrm{~nm}$-thick magnetostrictive film was deposited by electron beam physical vapor deposition and patterned on top of the microcantilever structure. The magnetostriction of the $\mathrm{Co}_{66} \mathrm{Fe}_{34}$ films was confirmed by measuring the deflection of the cantilevers under a varying magnetic field, reaching displacements up to $8 \mathrm{~nm}$. The configuration was simulated using COMSOL software, yielding a similar deflection behavior as a function of the magnetic field, with a film with a magnetostrictive coefficient of $\lambda_{\mathrm{S}} \sim 55$ p.p.m. The experimental configuration uses a laser and a position sensitive detector to measure the displacement, based on an optical lever configuration, and a piezoelectric stage to calibrate the system.
\end{abstract}

Keywords: Magnetostriction, Thin films, Micromechanical \& nanomechanical oscillators, Functional Materials, Evaporation, Bending

\section{Introduction}

Internet of things, the so-called IoT, and the Internet of Energy, the so-called IoE, are emerging in our daily lives through the development of new applications towards Smart Cities [1,2]. Such unscalable growth is fostering the market through the introduction of new sensor-enabled mobile and web applications almost every day [1]. A significant social impact is imminent, namely by allowing the emergence of IoT ecosystems supported by open technologies and platforms [1,2]. Although this tremendous evolution of IoT is being backed by new software, advances at a hardware level are still required. Thus, the demand for innovative multifunctional sensors in the near future is urgent, specifically sensors with new functionalities, and higher sensitivity [3].

The field of magnetic sensors/actuators is one of the fields that is being boosted in this new era and, although being studied for many years now, the combination of the magnetostrictive effect with microelectro-mechanical systems (MEMS) is still at an early stage. MEMS are being applied in many research

\footnotetext{
1 *Corresponding author: ampereira@fc.up.pt

Phone: + 351220402369

Fax: + 351220402369
} 
areas such as magnetic field sensing (high magnetic fields or ultra-low magnetic fields) [4,5], actuator systems for wireless devices [6], or biological applications, namely in platforms for mass and force detection [7]. In the ' $90 \mathrm{~s}$, Koch and co-authors [8] demonstrated the use of a cantilever beam technique as a potential magnetometer for the quantitative measurement of magnetization, magnetostriction, and magnetocrystalline anisotropy. It took one decade to demonstrate that an optical method could be implemented to detect the bending of a cantilever and that it could be achieved by depositing a magnetostrictive material on the cantilever [9]. Several efforts to combine magnetic systems and motion of miniaturized vibrating mechanical structures have been reported. Herein, research on magnetic material characteristics $[10,11]$ and sensitive detection of an external magnetic field $[12,13]$ should be highlighted. More recently, Park and co-authors [14] showed that by using Ni thin films, it is possible to tailor the resonant behavior of miniaturized mechanical systems by controlling the surface stress originated by the magnetostrictive film. The authors reported an effective magnetostriction of 260 p.p.m. at a low-saturation field of $\approx 10 \mathrm{mT}$ for an atomic composition close to $\mathrm{Co}: \mathrm{Fe}=66: 34$. In the literature, the atomic composition of $\mathrm{Co}: \mathrm{Fe}=66: 34$ is typically associated to be responsible for the highest magnetostrictive effect [15]. Electroplating [16] and DC sputtering [17] have been reported for the production of such thin films. Despite the achieved breakthrough, only a few works on thin film preparation of this promising intermetallic compound were subsequently performed.

Most magnetostrictive applications are based on polycrystalline or single crystal $\mathrm{Si}$ substrates $[14,16,18-20]$ and, to the best of our knowledge, no studies on magnetostrictive cantilevers using hydrogenated amorphous silicon (a-Si:H) as structural layer, which presents high potential for MEMS applications due to the low temperature process and reduced residual stress [21], have been published yet.

In the present work, the production of a magnetostrictive microcantilever based on a-Si: $\mathrm{H}$ and amorphous $\mathrm{Co}_{66} \mathrm{Fe}_{34}$ thin films are addressed and tested. Numerical simulations to support the results are performed, using a finite elements method.

\section{Experimental Details}

The surface and chemical composition of the produced films and MEMS devices were evaluated by Scanning Electron Microscopy (SEM) using Phillips-FEI/Quanta 400 FEG, from Materials Center of the University of Porto (CEMUP), with a coupled Energy Dispersive Spectroscopy (EDS). X-ray diffraction (XRD) measurements were carried out, at room temperature (RT), using a Rigaku Smartlab X-ray diffractometer in the Bragg-Brentano configuration, operating at $9 \mathrm{~kW}$, with the $K_{\alpha}$ line of Copper corresponding to a wavelength of $1.5418 \AA$. Magnetic properties of the bulk sample were obtained by measuring the M-H curve with a Vibrating Sample Magnetometer (VSM), KLA Tencor EV7, operating at $\mathrm{RT}$ and with an applied magnetic field varying in the range between $\pm 0.8 \mathrm{~T}$.

To measure the deflection of the free end of the microcantilevers as a function of the applied magnetic field, the microcantilevers were placed between two Helmholtz coils using a commonly implemented optical lever setup, as illustrated in Fig. 1.

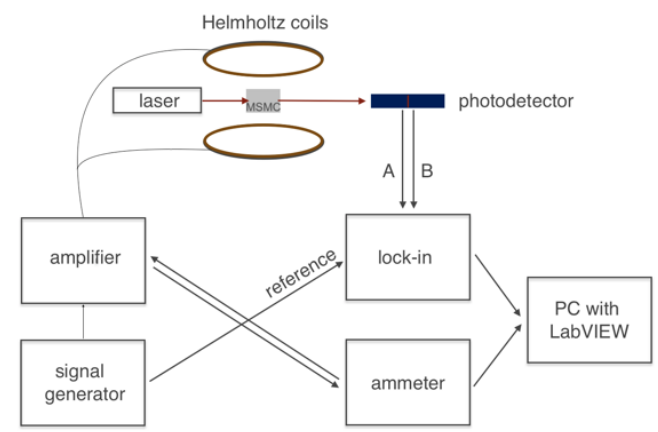

Fig. 1. Schematic representation of the measurement setup. An AC magnetic field is induced in the Helmholtz coils by amplifying a sinusoidal voltage signal from the signal generator. The varying magnetic field causes the microcantilevers to bend and the deflection is measured using a PSD connected to a lock-in, which in turn is connected to a computer.

The microcantilevers were placed so that the magnetic field was applied parallel to their length (longitudinal). A BOP 50-4D Kepco was used to amplify a sinusoidal signal from a signal generator (Agilent 33220A), which was injected into the coils, producing an AC magnetic field. A collimated laser source $(658 \mathrm{~nm} ; 10 \mu \mathrm{W})$ was used to shine light on a microcantilever tip, and an Eltec 404-4 position 
sensitive detector (PSD) was used to collect the reflected signal [15,22]. The laser beam was pointed at one microcantilever at a time. The varying magnetic field leads to the change in volume of the magnetostrictive thin film, resulting in the deflection of the microcantilevers. The amplitude of the measured signal, $\mathrm{R}$, and the phase between the signal measured in the photodetector and the reference signal from the signal generator, $\phi$, were obtained by a lock-in (Stanford Research Systems SR830), which was connected to the photodetector. Since the AC current in the coils induces a vibrating movement on the microcantilevers, the lock-in was set to be driven at twice the reference frequency to measure the second harmonic. The integration time of the signal ranged from $1 \mathrm{~s}$ to $30 \mathrm{~s}$. A computer with LabVIEW was used for data acquisition. To convert the values of R into displacement, a PSI-5A4E piezoelectric (PZT) sheet was placed beneath the microcantilevers and the PZT-induced motion of the non-actuated cantilever was individually calibrated. The calibration was performed by applying an increasing voltage between the upper and bottom surfaces of the PZT, and measuring the amplitude R of the first harmonic in the lock-in. The values for the applied magnetic field were measured with a Hall probe.

\section{Microfabrication of Magnetostrictive Cantilevers}

\subsection{Magnetostrictive $\mathrm{Co}_{66} \mathrm{Fe}_{34}$ Thin Film Deposition}

The $\mathrm{Co}_{66} \mathrm{Fe}_{34}$ target material was produced by arc melting using as starting reagents Alfa Aesar Co pieces with $99.9+\%$ purity and Fe pieces with $99.97+\%$ purity. After several high-vacuum purges, an Argon working pressure of 700 mbar was used for the arc-melting of the starting reagents. Electron Beam Physical Vapor Deposition (EB-PVD) from Edwards Auto 306 system was used to produce the $\mathrm{Co}_{66} \mathrm{Fe}_{34}$ films. A $10 \mathrm{~mA}$ current and a $4 \mathrm{kV}$ voltage were applied on a Tungsten filament. The kinetic energy of the generated electron beam is converted into thermal energy that heats the target located underneath. The procedure adopted in this work was of small deposition time intervals of 3 minutes, in a total of 72 minutes, with 5minute intervals between each deposition to avoid overheating. Magnetostrictive thin films were achieved with thicknesses up to $136 \pm 5 \mathrm{~nm}$.

\subsection{Fabrication of Microcantilevers with Magnetostrictive Film}

The microcantilevers were fabricated by surface-micromachining using a 4-mask process. The fabrication started with the cleaning of the substrate, a $0.7 \mathrm{~mm}$-thick glass (Corning 1737), followed by the deposition of a $1.02 \mu \mathrm{m}$-thick Al sacrificial layer using a Nordiko 7000 DC magnetron sputtering system. Optical lithography was performed by direct write laser (DWL) using a positive photoresist (PFR 7790G$27 \mathrm{cP}$, from JSR Micro), followed by wet etching (Gravure Aluminium Etchant Micropur MOS from Technic, France). Then, the structural layer of the microcantilevers, an $850 \mathrm{~nm}$-thick layer of n-doped hydrogenated amorphous silicon $(\mathrm{n}+\mathrm{a}-\mathrm{Si}: \mathrm{H})$, was deposited by plasma enhanced chemical vapor deposition, defined using DWL and patterned by reactive ion etching [23]. Another lithography process was performed on top of the cantilevers to define the region where the magnetostrictive film was to be deposited.

The $\mathrm{Co}_{66} \mathrm{Fe}_{34}$ film was deposited by EBPVD using intermittent deposition time intervals to avoid overheating. This methodology was chosen based on previous and already optimized tests. This step was followed by liftoff in Microstrip 3001 (Fujifilm). In order to protect the magnetostrictive film during the microcantilever release, the last lithography step was performed in order to define photoresist rectangles on top of the $\mathrm{Co}_{66} \mathrm{Fe}_{34}$ regions. Finally, the release of the microcantilevers was achieved using $\mathrm{Al}$ etchant in order to remove the Al sacrificial layer. After release, the dies were sequentially washed in water (to remove the $\mathrm{Al}$ etchant), acetone (to remove the photoresist), isopropanol and n-hexane (solvents with decreasing surface tension, to avoid stiction), and let dry at ambient temperature. 


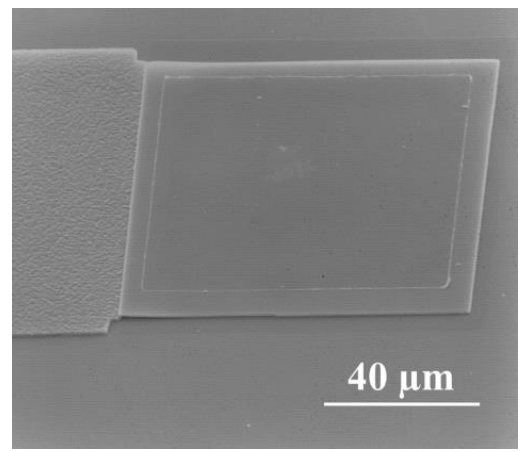

Fig. 2. Top-view SEM micrograph illustrating an example of the $\mathrm{Co}_{66} \mathrm{Fe}_{34}$ thin film deposited on top of the micrometric cantilever throughout an area smaller than the cantilever area.

\section{Results and Discussion}

\subsection{Magnetostrictive Microcantilevers}

A proof-of-concept experiment was undertaken to demonstrate the magnetostrictive behavior of the $\mathrm{Co}_{66} \mathrm{Fe}_{34}$ thin films, by depositing a layer of this material over a microstructure (Fig. 2a) and b)).

a)

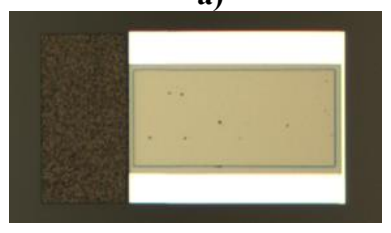

b)

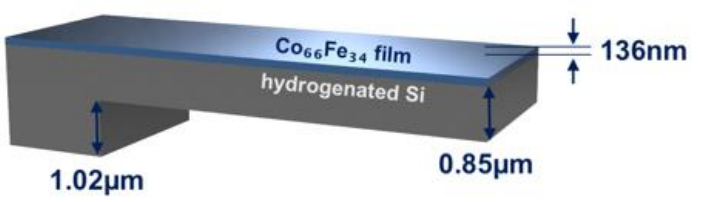

Fig. 2. Magnetostrictive microcantilever (MSMC): a) top view optical microscopy image and b) schematic illustration of the lateral view.

In the first attempt to integrate magnetostrictive films with thin-film silicon cantilevers, the magnetostrictive thin films were deposited by EBPVD using a continuous 80 min deposition. However, as observed in Fig. 3, the high temperature reached by the magnetostrictive material during its deposition degraded the microcantilever. This degradation can be associated with the higher thermal expansion coefficient of $\mathrm{Al}$ in comparison to those of silica and $\mathrm{Co}_{66} \mathrm{Fe}_{34}$.

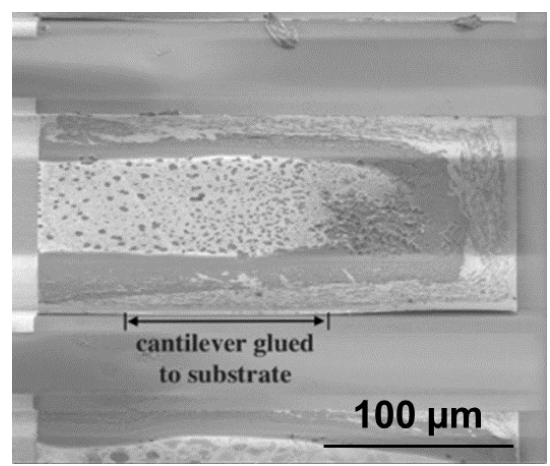

Fig. 3. Top-view SEM imaging shows a damaged microcantilever in contact with the substrate (stiction). 
To overcome the destruction of the microcantilevers caused by overheating during the deposition of the magnetostrictive material, the process was conducted with intermittent deposition times followed by a waiting period. Thus, the thin films were deposited using small time intervals of 3 minutes, in a total of 72 minutes, with 5-minute intervals between each deposition. Using a Dektak XT profilometer from Bruker, a thin film thickness of approximately $136 \pm 5 \mathrm{~nm}$ was achieved.

In Figure 4, the XRD pattern of the MSMC is depicted.

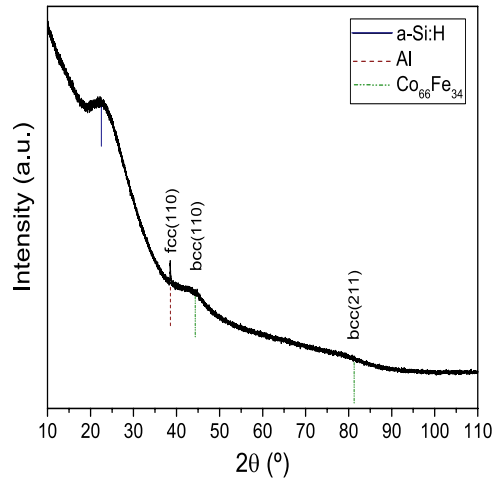

Fig. 4. The X-ray diffractogram for the MSMC measured at RT showing substantially broad peaks, associated with a non-crystalline $\mathrm{C}_{66} \mathrm{Fe}_{34}$ layer over the microcantilevers (linear intensity for better reading of the plot).

The XRD was performed on the MSMCs before removing the photoresist and the Al sacrificial layer. The first broad band at $2 \theta \approx 23^{\circ}-26^{\circ}$ is indexed to amorphous/nanocrystalline $\mathrm{n}^{+} \mathrm{a}-\mathrm{Si}: \mathrm{H}$ layer [24]. Additional Bragg reflections are present at $2 \theta \approx 38^{\circ}$ corresponding to the $\mathrm{Al} \mathrm{fcc}(110)$ phase [25], and at $2 \theta \approx 45^{\circ}$ and $2 \theta \approx 83^{\circ}$ corresponding to the $\operatorname{bcc}(110)$ and $\operatorname{bcc}(211)$ planes of the phase, respectively, indicating the presence of the Co-Fe compound [26]. Nonetheless, the last referenced peaks are broad and weak, which is associated with a lack of a long-range crystalline organization characteristic of the amorphous/nanocrystalline structure.

\subsection{Displacement Measurements}

As proof of concept, the presence of a magnetostrictive effect was confirmed by magnetically actuating the MSMC movement using the configuration illustrated in Fig. 1.

Fig. 5 depicts the displacement for the microcantilever $M_{1}(100 \mu \mathrm{m} \times 60 \mu \mathrm{m})$ as a function of the amplitude of AC magnetic field. It is possible to observe a parabolic increase of displacement with the applied magnetic field. To test the repeatability of the deflection method using this characterization setup, two measurements were carried out using $M_{1}$ under the same conditions and for the same frequency. The observed shift on the displacement curves its minimal, which indicates that the microcantilever was not critically damaged for such low fields and that it returns to its initial geometric configuration after the magnetic field is removed.

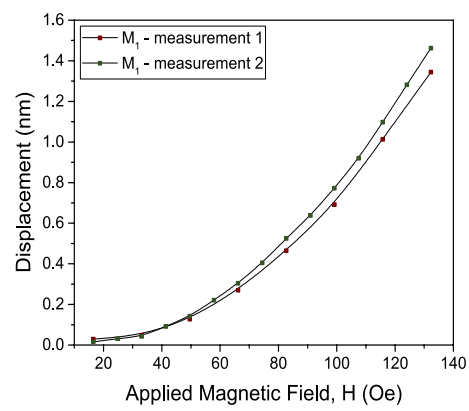

Fig. 5. Displacement as a function of the applied magnetic field for microcantilever $\mathrm{M}_{1}$ was obtained using the configuration schematized in Fig. 1. Nanometric displacements were measured over the magnetic field range considered, and the results show acceptable repeatability. 
In order to have an estimation of the magnetostrictive coefficient of the $\mathrm{Co}_{66} \mathrm{Fe}_{34}$ thin film, numerical simulations were performed with the COMSOL Multiphysics software. By combining the Structural Mechanics and the AC/DC modules of the software, the cantilever $\mathrm{M}_{1}$ was simulated inside a box of air. The Young's modulus of the $\mathrm{n}^{+} \mathrm{a}-\mathrm{Si}: \mathrm{H}$ and $\mathrm{Co}_{66} \mathrm{Fe}_{34}$ were set to $80 \mathrm{GPa}$ [27] and $140 \mathrm{GPa}$ [15], respectively. Since it was not possible to measure the magnetic properties of the films deposited on the microcantilevers (as it would critically damage the microstructure), the magnetic behavior of the ferromagnetic film was considered to be similar to that of the amorphous CoFeB film reported by Jen and Yao [28]. An isotropic model for the magnetostrictive effect was used, and the coefficient was varied to fit the experimental results.

The best resemble simulation is depicted in Fig. 6 and the corresponding coefficient obtained was $\lambda_{\mathrm{S}}=$ $55 \times 10^{-6}(55$ p.p.m.). The achieved coefficient is in good agreement with the literature. For instance, Cooke [29] and Hunter [15] groups reported about 80 p.p.m. in as-deposited thin films of $\mathrm{Co}_{66} \mathrm{Fe}_{34}$. In comparison with annealed $\mathrm{Co}_{66} \mathrm{Fe}_{34}$ thin films coefficient, the obtained value is low, however this was expected for the nanocrystalline/amorphous nature of the thin film here reported.

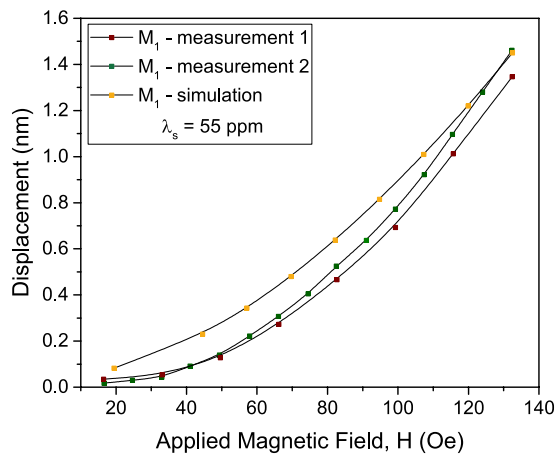

Fig. 6. Results from the simulation performed with COMSOL Multiphysics software and comparison with experimental data. This study allows us to conclude that the magnetostrictive coefficient of the $\mathrm{Co}_{6} \mathrm{Fe}_{34}$ film deposited on $\mathrm{n}+\mathrm{a}-\mathrm{Si}: \mathrm{H}$ is around $55 \times 10^{-6}(55$ p.p.m. $)$.

\section{Conclusions}

A microcantilever structure was developed using micro-fabrication techniques to verify magnetostriction in amorphous $\mathrm{Co}_{66} \mathrm{Fe}_{34}$ thin films. The optical characterization system based on the reflection of laser light on the surface of the MSMC allowed for the measurement of microcantilever nano-displacements, for applied magnetic fields ranging from $[0 ; 140]$ Oe, verifying magnetostriction on amorphous $\mathrm{Co}_{66} \mathrm{Fe}_{34}$ films. Numerical simulations performed with COMSOL Multiphysics software have allowed an estimation of the magnetostrictive coefficient of the $\mathrm{MSMC} \mathrm{M}_{1}$ up to 55 p.p.m., which is in agreement with the literature. In the future, an additional buffer layer needs to be implemented to minimize the influence of the thermal expansion.

\section{Acknowledgment}

This work was financially supported by Fundação para a Ciência e a Tecnologia (FCT)/MEC and FEDER under Program PT2020 through the projects UID/NAN/50024/2019, and NORTE-01-0145-FEDER022096 from NECL. A.M.P. and J.A.S. acknowledge ANI and FCT through the project POCI-01-0247FEDER-033783. J.H.B acknowledges the Grant SFRH/BD/88440/2012, the project PTDC/FIS$\mathrm{MAC} / 31302 / 2017$ and his contract DL57/2016 reference SFRH-BPD-87430/2012. R.M.R.P. (PD/BD/113960/2015) acknowledges FCT for a PhD Grant, through the research project uMEMS (PTDC/CTM-NAN/5052/2014), and for funding through the Research Unit IN-Institute of Nanoscience and Nanotechnology (UID/NAN/50024/2013).

\section{References}

[1] D. Miorandi, S. Sicari, F. De Pellegrini, I. Chlamtac, I Ad Hoc Networks. 10 (2012) 1497-1516.

[2] T. Islam, S.C. Mukhopadhyay, N.K. Suryadevara, IEEE Sens. J. 17 (2017) 577-584. 
[3] A. Rayes, S. Salam, Springer International Publishing, Cham, 2019.

[4] F. Keplinger, S. Kvasnica, H. Hauser, R. Grossinger, IEEE Trans. Magn. 39 (2003) 3304-3306.

[5] A. Guedes, G. Jaramillo, C. Buffa, G. Vigevani, S. Cardoso, D.C. Leitao, P.P. Freitas, D.A. Horsley, IEEE Trans. Magn. 48 (2012) 4115-4118.

[6] B.A. Cetiner, H. Jafarkhani, Jiang-Yuan Qian, Hui Jae Yoo, A. Grau, F. De Flaviis, IEEE Commun. Mag. 42 (2004) 62-70.

[7] X. Li, D.W. Lee, Meas. Sci. Technol. 23 (2012) 029501.

[8] M. Weber, R. Koch, K.H. Rieder, Phys. Rev. Lett. 73 (1994) 1166-1169.

[9] D. Sander, J. Kirschner, Appl. Phys. A. 87 (2007) 419-425.

[10] G. Wu, R. Zhang, X. Li, N. Zhang, J. Appl. Phys. 110 (2011) 124103.

[11] X.W. Dong, Y.J. Wu, J.G. Wan, T. Wei, Z.H. Zhang, S. Chen, H. Yu, J.M. Liu, J. Phys. D. Appl. Phys. 41 (2008) 035003.

[12] T. Nan, Y. Hui, M. Rinaldi, N.X. Sun, Sci. Rep. 3 (2013) 1985.

[13] B. Gojdka, R. Jahns, K. Meurisch, H. Greve, R. Adelung, E. Quandt, R. Knöchel, F. Faupel, Appl. Phys. Lett. 99 (2011) 223502.

[14] Y. Park, E. Lee, T. Kouh, Micromachines. 8 (2017) 109.

[15] D. Hunter, W. Osborn, K. Wang, N. Kazantseva, J. Hattrick-Simpers, R. Suchoski, R. Takahashi, M.L. Young, A. Mehta, L. Bendersky, S.E. Lofland, M. Wuttig, I. Takeuchi, Nat. Commun. 2 (2011) 518.

[16] B. Özkale, N. Shamsudhin, T. Bugmann, B.J. Nelson, S. Pané, Electrochem. Commun. 76 (2017) $15-19$.

[17] J. Zhou, S. Chen, W. Lin, Q. Qin, L. Liu, S. He, J. Chen, J. Magn. Magn. Mater. 441 (2017) 264270.

[18] L. Fu, S. Li, K. Zhang, I.H. Chen, V. Petrenko, Z. Cheng, Sensors. 7 (2007) 2929-2941.

[19] G. Engdahl, E. Quandt, Handbook of Giant Magnetostrictive Materials, 2000.

[20] Y.Y. Kim, Y.E. Kwon, Ultrasonics. 62 (2015) 3-19.

[21] J. Mouro, A. Gualdino, V. Chu, J.P. Conde, J. Appl. Phys. 114 (2013) 184905.

[22] G. Meyer, N.M. Amer, Appl. Phys. Lett. 53 (1988) 1045-1047.

[23] J. Mouro, V. Chu, J.P. Conde, J. Appl. Phys. 119 (2016) 154501.

[24] T.V. Torchynska, Phys. E Low-Dimensional Syst. Nanostructures. 44 (2011) 56-61.

[25] S.W. Lin, J.Y. Wu, S.D. Lin, M.C. Lo, M.H. Lin, C.T. Liang, Jpn. J. Appl. Phys. 52 (2013) 045801.

[26] T. Nakajima, T. Takeuchi, I. Yuito, K. Kato, M. Saito, K. Abe, T. Sasaki, T. Sekiguchi, S. Yamaura, Mater. Trans. 55 (2014) 556-560.

[27] L.B. Freund, S. Suresh, Thin film materials, 2004.

[28] S.U. Jen, Y.D. Yao, Y.T. Chen, J.M. Wu, C.C. Lee, T.L. Tsai, Y.C. Chang, J. Appl. Phys. 99 (2006) 053701 .

[29] M.D. Cooke, M.R.J. Gibbs, R.F. Pettifer, J. Magn. Magn. Mater. 237 (2001) 175-180. 